\title{
Rotational Spectrum, Tunneling Motions and Intramolecular Potential Barriers in Benzyl Mercaptan
}

Rizalina Tama Saragi,,$^{\dagger}$ Marcos Juanes, ${ }^{\dagger}$ Walther Caminati, ${ }^{*}+,+\neq$ Alberto Lesarri, ${ }^{*},+$ Lourdes Enríquez, ${ }^{\S}$ Martín Jaraíz ${ }^{\S}$

†Departamento de Química Física y Química Inorgánica - IU CINQUIMA, Facultad de Ciencias, Universidad de Valladolid, Paseo de Belén, 7, E-47011, Valladolid, Spain,

§Departamento de Electrónica, ETSIT, Universidad de Valladolid, Paseo de Belén, 11, E-47011, Valladolid, Spain.

\$Permanent address: Via della Cavriola, 20, Bologna, Italy 
Table S1. Predicted rotational parameters for benzyl mercaptan using MP2 and the alternative basis sets 6-311++G(3df,3pd) and aug-cc-pVTZ.

\begin{tabular}{lcc}
\hline & $\begin{array}{c}\text { MP2/ } \\
\text { 6-311++G(3df,3pd) }\end{array}$ & $\begin{array}{c}\text { MP2/ } \\
\text { aug-cc-pVTZ }\end{array}$ \\
\hline$A_{e} / \mathrm{MHz}^{\mathrm{a}}$ & $4143.71[-0.6 \%]$ & $4141.03[-0.6 \%]$ \\
$B_{e} / \mathrm{MHz}$ & $1015.96[1.4 \%]$ & $1012.13[1.0 \%]$ \\
$C_{e} / \mathrm{MHz}$ & $903.84[1.3 \%]$ & $900.90[1.0 \%]$ \\
$D_{J} / \mathrm{kHz}$ & 0.1425 & 0.1420 \\
$D_{J K} / \mathrm{kHz}$ & 1.7756 & 2.0261 \\
$D_{K} / \mathrm{kHz}$ & 1.7346 & 1.5078 \\
$d_{1} / \mathrm{kHz}$ & 0.0047 & 0.0048 \\
$d_{2} / k \mathrm{~Hz}$ & 0.0052 & 0.0059 \\
$\left|\mu_{a}\right| / \mathrm{D}$ & 1.2 & 1.2 \\
$\left|\mu_{b}\right| / \mathrm{D}$ & 0.6 & 0.6 \\
$\mu_{c} \mid / \mathrm{D}$ & 0.5 & 0.5 \\
$\mathrm{SC} \alpha-\mathrm{C} 1 \mathrm{C} 2 / \mathrm{deg}$ & 76.2 & 76.4 \\
$\mathrm{HS}-\mathrm{C} \alpha \mathrm{C} 1 /$ deg & 52.0 & 52.3 \\
\hline aRotational constants $(A, B, C)$, Watson's S-reduction centrifugal distortion constants \\
$\left(D_{J}, D_{J K}, D_{K}, d_{1}, d_{2}\right)$, electric dipole moments $\left(\mu_{a}, \alpha=\mathrm{a}, \mathrm{b}, \mathrm{c}\right)$ and structural parameters \\
of the thiol group.
\end{tabular}


Table S2. Rotational transitions of benzyl mercaptan (parent species) and differences between the observed and calculated frequencies (o-c) for the fit of Table 2.

\begin{tabular}{|c|c|c|c|c|c|c|c|c|c|c|}
\hline & $J^{\prime}$ & $K_{-_{-}^{\prime}}^{\prime}$ & $K_{+l^{\prime}}$ & $v^{\prime}$ & $J^{\prime \prime}$ & $K_{-I^{\prime \prime}}$ & $K_{+1}{ }^{\prime \prime}$ & $v^{\prime \prime}$ & Freq. / MHz & $\mathrm{o}-\mathrm{c} / \mathrm{kHz}$ \\
\hline 1 & 2 & 1 & 2 & 1 & 2 & 0 & 2 & 1 & 3084.1594 & -0.0013 \\
\hline 2 & 2 & 1 & 2 & 0 & 2 & 0 & 2 & 0 & 3085.1389 & 0.0031 \\
\hline 3 & 4 & 2 & 2 & 1 & 5 & 1 & 5 & 0 & 3091.0826 & -0.0037 \\
\hline 4 & 7 & 1 & 6 & 0 & 6 & 2 & 5 & 1 & 3075.4514 & 0.0164 \\
\hline 5 & 8 & 1 & 8 & 1 & 7 & 2 & 6 & 1 & 3171.7384 & -0.0121 \\
\hline 6 & 1 & 1 & 1 & 0 & 1 & 0 & 1 & 0 & 3176.7785 & 0.0103 \\
\hline 7 & 1 & 1 & 1 & 1 & 1 & 0 & 1 & 1 & 3177.3150 & -0.0038 \\
\hline 8 & 8 & 1 & 7 & 0 & 8 & 0 & 8 & 1 & 3450.4312 & -0.0284 \\
\hline 9 & 2 & 1 & 2 & 1 & 1 & 1 & 1 & 1 & 3670.1654 & -0.0039 \\
\hline 10 & 2 & 1 & 2 & 0 & 1 & 1 & 1 & 0 & 3688.5765 & -0.0111 \\
\hline 11 & 2 & 0 & 2 & 1 & 1 & 0 & 1 & 1 & 3763.3311 & 0.0036 \\
\hline 12 & 2 & 0 & 2 & 0 & 1 & 0 & 1 & 0 & 3780.2167 & -0.0033 \\
\hline 13 & 3 & 2 & 2 & 1 & 4 & 1 & 3 & 0 & 3783.2465 & 0.0011 \\
\hline 14 & 4 & 0 & 4 & 1 & 3 & 1 & 2 & 1 & 3882.3999 & 0.0092 \\
\hline 15 & 2 & 1 & 1 & 1 & 1 & 1 & 0 & 1 & 3886.0464 & -0.0057 \\
\hline 16 & 2 & 1 & 1 & 0 & 1 & 1 & 0 & 0 & 3887.9522 & -0.0109 \\
\hline 17 & 4 & 0 & 4 & 0 & 3 & 1 & 2 & 0 & 3988.9329 & -0.0089 \\
\hline 18 & 9 & 1 & 8 & 0 & 9 & 0 & 9 & 1 & 4219.8872 & -0.0230 \\
\hline 19 & 5 & 0 & 5 & 0 & 4 & 1 & 4 & 1 & 4498.7585 & -0.0038 \\
\hline 20 & 9 & 1 & 9 & 1 & 8 & 2 & 7 & 1 & 4507.1821 & -0.0062 \\
\hline 21 & 2 & 1 & 2 & 0 & 1 & 0 & 1 & 1 & 4692.5096 & -0.0008 \\
\hline 22 & 3 & 0 & 3 & 1 & 2 & 1 & 2 & 0 & 4703.8139 & -0.0029 \\
\hline 23 & 3 & 2 & 1 & 1 & 4 & 1 & 4 & 0 & 4755.8644 & 0.0133 \\
\hline 24 & 7 & 1 & 6 & 1 & 6 & 2 & 4 & 1 & 4842.9402 & -0.0053 \\
\hline 25 & 6 & 1 & 5 & 1 & 5 & 2 & 4 & 0 & 4894.2989 & 0.0128 \\
\hline 26 & 7 & 1 & 6 & 0 & 6 & 2 & 4 & 0 & 5022.4820 & -0.0122 \\
\hline 27 & 10 & 1 & 9 & 0 & 10 & 0 & 10 & 1 & 5106.9634 & 0.0133 \\
\hline 28 & 1 & 1 & 0 & 0 & 0 & 0 & 0 & 0 & 5168.9131 & 0.0042 \\
\hline 29 & 1 & 1 & 0 & 1 & 0 & 0 & 0 & 1 & 5169.4362 & 0.0033 \\
\hline 30 & 8 & 1 & 7 & 0 & 7 & 2 & 6 & 1 & 5341.3821 & 0.0325 \\
\hline 31 & 5 & 0 & 5 & 1 & 4 & 1 & 3 & 1 & 5427.1791 & 0.0068 \\
\hline 32 & 1 & 1 & 0 & 1 & 1 & 0 & 1 & 0 & 5458.3563 & 0.0011 \\
\hline 33 & 3 & 2 & 1 & 1 & 4 & 0 & 4 & 1 & 5483.3809 & -0.0270 \\
\hline 34 & 3 & 1 & 3 & 1 & 2 & 1 & 2 & 1 & 5507.5432 & -0.0057 \\
\hline 35 & 3 & 1 & 3 & 0 & 2 & 1 & 2 & 0 & 5533.9168 & -0.0078 \\
\hline 36 & 9 & 4 & 5 & 1 & 10 & 3 & 8 & 0 & 5576.5731 & 0.0186 \\
\hline 37 & 2 & 1 & 1 & 1 & 2 & 0 & 2 & 0 & 5564.1947 & 0.0074 \\
\hline 38 & 3 & 0 & 3 & 1 & 2 & 0 & 2 & 1 & 5633.0005 & 0.0007 \\
\hline 39 & 5 & 0 & 5 & 0 & 4 & 1 & 3 & 0 & 5637.3747 & -0.0095 \\
\hline 40 & 3 & 0 & 3 & 0 & 2 & 0 & 2 & 0 & 5663.0485 & -0.0072 \\
\hline 41 & 3 & 2 & 2 & 1 & 2 & 2 & 1 & 1 & 5666.0084 & -0.0040 \\
\hline 42 & 3 & 2 & 1 & 1 & 2 & 2 & 0 & 1 & 5675.9207 & -0.0022 \\
\hline 43 & 3 & 2 & 2 & 0 & 2 & 2 & 1 & 0 & 5679.0714 & -0.0096 \\
\hline 44 & 3 & 2 & 1 & 0 & 2 & 2 & 0 & 0 & 5687.6666 & -0.0077 \\
\hline 45 & 3 & 1 & 2 & 1 & 3 & 0 & 3 & 0 & 5729.6532 & 0.0095 \\
\hline 46 & 3 & 1 & 2 & 0 & 2 & 1 & 1 & 0 & 5826.2286 & -0.0088 \\
\hline 47 & 3 & 1 & 2 & 1 & 2 & 1 & 1 & 1 & 5828.5087 & -0.0034 \\
\hline 48 & 12 & 2 & 11 & 0 & 12 & 1 & 11 & 0 & 5841.3469 & -0.0061 \\
\hline 49 & 2 & 2 & 1 & 1 & 3 & 1 & 2 & 0 & 5868.7797 & 0.0088 \\
\hline 50 & 4 & 1 & 3 & 1 & 4 & 0 & 4 & 0 & 5961.8866 & 0.0109 \\
\hline
\end{tabular}




\begin{tabular}{|c|c|c|c|c|c|c|c|c|c|c|}
\hline 51 & 5 & 1 & 5 & 0 & 4 & 1 & 3 & 1 & 6083.5674 & -0.0001 \\
\hline 52 & 8 & 2 & 6 & 0 & 8 & 1 & 7 & 1 & 6199.7684 & 0.0160 \\
\hline 53 & 4 & 1 & 3 & 0 & 3 & 1 & 3 & 1 & 6215.1865 & -0.0128 \\
\hline 54 & 5 & 1 & 4 & 1 & 5 & 0 & 5 & 0 & 6269.4565 & 0.0124 \\
\hline 55 & 11 & 2 & 10 & 0 & 11 & 1 & 10 & 0 & 6356.2023 & 0.0004 \\
\hline 56 & 7 & 2 & 5 & 0 & 7 & 1 & 6 & 1 & 6396.7655 & 0.0103 \\
\hline 57 & 3 & 1 & 3 & 0 & 2 & 0 & 2 & 1 & 6463.0994 & -0.0082 \\
\hline 58 & 2 & 2 & 0 & 1 & 3 & 1 & 3 & 0 & 6463.5170 & 0.0022 \\
\hline 59 & 6 & 0 & 6 & 0 & 5 & 1 & 5 & 1 & 6503.7367 & 0.0035 \\
\hline 60 & 6 & 2 & 4 & 0 & 6 & 1 & 5 & 1 & 6608.4563 & 0.0081 \\
\hline 61 & 4 & 0 & 4 & 1 & 3 & 1 & 3 & 0 & 6656.0396 & 0.0098 \\
\hline 62 & 6 & 1 & 5 & 1 & 6 & 0 & 6 & 0 & 6661.8549 & 0.0087 \\
\hline 63 & 5 & 2 & 3 & 0 & 5 & 1 & 4 & 1 & 6822.1413 & 0.0043 \\
\hline 64 & 6 & 1 & 6 & 0 & 5 & 1 & 4 & 1 & 6832.6853 & 0.0212 \\
\hline 65 & 10 & 2 & 9 & 0 & 10 & 1 & 9 & 0 & 6846.1945 & -0.0099 \\
\hline 66 & 5 & 3 & 2 & 1 & 6 & 2 & 5 & 0 & 6919.8106 & -0.0195 \\
\hline 67 & 8 & 1 & 7 & 1 & 7 & 2 & 5 & 1 & 6987.6276 & -0.0210 \\
\hline 68 & 4 & 2 & 2 & 0 & 4 & 1 & 3 & 1 & 7023.7019 & 0.0060 \\
\hline 69 & 10 & 2 & 9 & 1 & 10 & 1 & 9 & 1 & 7033.8647 & 0.0191 \\
\hline 70 & 7 & 1 & 6 & 1 & 6 & 2 & 5 & 0 & 7110.5194 & 0.0134 \\
\hline 71 & 8 & 1 & 7 & 0 & 7 & 2 & 5 & 0 & 7143.4084 & 0.0062 \\
\hline 72 & 7 & 1 & 6 & 1 & 7 & 0 & 7 & 0 & 7148.8124 & 0.0038 \\
\hline 73 & 2 & 1 & 1 & 0 & 1 & 0 & 1 & 0 & 7165.3076 & 0.0012 \\
\hline 74 & 2 & 1 & 1 & 1 & 1 & 0 & 1 & 1 & 7171.5623 & 0.0002 \\
\hline 75 & 6 & 4 & 2 & 0 & 7 & 3 & 5 & 1 & 7196.6515 & -0.0137 \\
\hline 76 & 3 & 2 & 1 & 0 & 3 & 1 & 2 & 1 & 7200.1651 & 0.0078 \\
\hline 77 & 12 & 1 & 11 & 0 & 12 & 0 & 12 & 1 & 7225.4400 & 0.0032 \\
\hline 78 & 1 & 1 & 1 & 1 & 0 & 0 & 0 & 0 & 7241.7273 & -0.0024 \\
\hline 79 & 6 & 0 & 6 & 0 & 5 & 1 & 4 & 0 & 7258.9888 & 0.0050 \\
\hline 80 & 9 & 2 & 8 & 0 & 9 & 1 & 8 & 0 & 7302.3173 & 0.0309 \\
\hline 81 & 2 & 2 & 0 & 0 & 2 & 1 & 1 & 1 & 7341.0072 & 0.0121 \\
\hline 82 & 4 & 1 & 4 & 1 & 3 & 1 & 3 & 1 & 7353.8158 & -0.0054 \\
\hline 83 & 4 & 1 & 4 & 0 & 3 & 1 & 3 & 0 & 7383.5821 & -0.0045 \\
\hline 84 & 9 & 2 & 8 & 1 & 9 & 1 & 8 & 1 & 7408.4616 & -0.0206 \\
\hline 85 & 4 & 0 & 4 & 1 & 3 & 0 & 3 & 1 & 7486.1411 & 0.0034 \\
\hline 86 & 6 & 0 & 6 & 1 & 5 & 1 & 4 & 1 & 7490.1258 & 0.0121 \\
\hline 87 & 4 & 0 & 4 & 0 & 3 & 0 & 3 & 0 & 7537.2047 & -0.0052 \\
\hline 88 & 4 & 2 & 3 & 1 & 3 & 2 & 2 & 1 & 7553.5880 & -0.0013 \\
\hline 89 & 4 & 3 & 2 & 1 & 3 & 3 & 1 & 1 & 7559.0736 & 0.0107 \\
\hline 90 & 4 & 3 & 1 & 1 & 3 & 3 & 0 & 1 & 7559.2456 & -0.0018 \\
\hline 91 & 4 & 2 & 3 & 0 & 3 & 2 & 2 & 0 & 7571.5093 & -0.0108 \\
\hline 92 & 4 & 3 & 2 & 0 & 3 & 3 & 1 & 0 & 7575.2816 & 0.0088 \\
\hline 93 & 4 & 3 & 1 & 0 & 3 & 3 & 0 & 0 & 7575.3915 & -0.0176 \\
\hline 94 & 4 & 2 & 2 & 1 & 3 & 2 & 1 & 1 & 7578.2992 & 0.0018 \\
\hline 95 & 4 & 2 & 2 & 0 & 3 & 2 & 1 & 0 & 7592.9738 & -0.0067 \\
\hline 96 & 2 & 2 & 1 & 0 & 2 & 1 & 2 & 1 & 7662.9326 & 0.0122 \\
\hline 97 & 8 & 2 & 7 & 0 & 8 & 1 & 7 & 0 & 7715.3601 & -0.0124 \\
\hline 98 & 8 & 1 & 7 & 1 & 8 & 0 & 8 & 0 & 7739.2164 & -0.0071 \\
\hline 99 & 4 & 1 & 3 & 0 & 3 & 1 & 2 & 0 & 7751.5290 & -0.0089 \\
\hline 100 & 4 & 1 & 3 & 1 & 3 & 1 & 2 & 1 & 7769.4383 & -0.0036 \\
\hline 101 & 8 & 2 & 7 & 1 & 8 & 1 & 7 & 1 & 7810.1925 & -0.0306 \\
\hline 102 & 3 & 2 & 2 & 0 & 3 & 1 & 3 & 1 & 7834.4612 & 0.0089 \\
\hline
\end{tabular}


Table S3. Rotational transitions of the ${ }^{34} \mathrm{~S}$ isotopologue of benzyl mercaptan and differences between the observed and calculated frequencies (o-c) for the fit of Table 2.

\begin{tabular}{|c|c|c|c|c|c|c|c|c|c|c|}
\hline & $J^{\prime}$ & $K_{-I}$ & $K_{+1}^{\prime}$ & $v^{\prime}$ & $J^{\prime \prime}$ & $K_{-1}^{\prime \prime}$ & $K_{+1}^{\prime \prime}$ & $v^{\prime \prime}$ & Freq. / $\mathrm{MHz}$ & $O-c / M H z$ \\
\hline 1 & 2 & 1 & 2 & $\overline{1}$ & 1 & 1 & 1 & 1 & 3581.5585 & -0.0070 \\
\hline 2 & 2 & 1 & 2 & 0 & 1 & 1 & 1 & 0 & 3597.3278 & -0.0012 \\
\hline 3 & 2 & 0 & 2 & 1 & 1 & 0 & 1 & 1 & 3671.1601 & -0.0109 \\
\hline 4 & 2 & 0 & 2 & 0 & 1 & 0 & 1 & 0 & 3685.5767 & -0.0062 \\
\hline 5 & 2 & 1 & 1 & 1 & 1 & 1 & 0 & 1 & 3785.3724 & 0.0028 \\
\hline 6 & 2 & 1 & 1 & 0 & 1 & 1 & 0 & 0 & 3787.0562 & -0.0014 \\
\hline 7 & 1 & 1 & 0 & 0 & 0 & 0 & 0 & 0 & 5128.6650 & -0.0009 \\
\hline 8 & 1 & 1 & 0 & 1 & 0 & 0 & 0 & 1 & 5129.1973 & 0.0086 \\
\hline 9 & 3 & 1 & 3 & 1 & 2 & 1 & 2 & 1 & 5374.6609 & -0.0072 \\
\hline 10 & 3 & 1 & 3 & 0 & 2 & 1 & 2 & 0 & 5395.9312 & 0.0061 \\
\hline 11 & 3 & 0 & 3 & 1 & 2 & 0 & 2 & 1 & 5496.9085 & -0.0253 \\
\hline 12 & 3 & 0 & 3 & 0 & 2 & 0 & 2 & 0 & 5521.8784 & -0.0088 \\
\hline 13 & 3 & 2 & 2 & 1 & 2 & 2 & 1 & 1 & 5524.0345 & -0.0041 \\
\hline 14 & 3 & 2 & 1 & 1 & 2 & 2 & 0 & 1 & 5532.7903 & -0.0074 \\
\hline 15 & 3 & 2 & 2 & 0 & 2 & 2 & 1 & 0 & 5536.2336 & -0.0051 \\
\hline 16 & 3 & 2 & 1 & 0 & 2 & 2 & 0 & 0 & 5543.8480 & 0.0055 \\
\hline 17 & 3 & 1 & 2 & 0 & 2 & 1 & 1 & 0 & 5675.2128 & 0.0019 \\
\hline 18 & 3 & 1 & 2 & 1 & 2 & 1 & 1 & 1 & 5677.6857 & 0.0089 \\
\hline 19 & 4 & 1 & 4 & 1 & 3 & 1 & 3 & 1 & 7176.3366 & 0.0073 \\
\hline 20 & 4 & 1 & 4 & 0 & 3 & 1 & 3 & 0 & 7196.6574 & 0.0165 \\
\hline 21 & 4 & 0 & 4 & 1 & 3 & 0 & 3 & 1 & 7309.5400 & -0.0444 \\
\hline 22 & 4 & 0 & 4 & 0 & 3 & 0 & 3 & 0 & 7350.4405 & -0.0029 \\
\hline 23 & 4 & 2 & 3 & 1 & 3 & 2 & 2 & 1 & 7364.4515 & -0.0029 \\
\hline 24 & 4 & 2 & 3 & 0 & 3 & 2 & 2 & 0 & 7381.1712 & 0.0061 \\
\hline 25 & 4 & 2 & 2 & 1 & 3 & 2 & 1 & 1 & 7386.3021 & 0.0034 \\
\hline 26 & 4 & 2 & 2 & 0 & 3 & 2 & 1 & 0 & 7400.1732 & 0.0154 \\
\hline 27 & 4 & 1 & 3 & 0 & 3 & 1 & 2 & 0 & 7551.1437 & -0.0070 \\
\hline 28 & 4 & 1 & 3 & 1 & 3 & 1 & 2 & 1 & 7568.7333 & 0.0180 \\
\hline 29 & 1 & 1 & 0 & 0 & 0 & 0 & 0 & 0 & 5128.6650 & -0.0009 \\
\hline 30 & 1 & 1 & 0 & 1 & 0 & 0 & 0 & 1 & 5129.1973 & 0.0086 \\
\hline 31 & 2 & 1 & 1 & 0 & 1 & 0 & 1 & 0 & 7071.6379 & 0.0036 \\
\hline 32 & 2 & 1 & 1 & 1 & 1 & 0 & 1 & 1 & 7077.0935 & 0.0010 \\
\hline 33 & 4 & 1 & 3 & 1 & 4 & 0 & 4 & 0 & 5842.2133 & 0.0136 \\
\hline 34 & 2 & 1 & 1 & 1 & 2 & 0 & 2 & 0 & 5468.1251 & -0.0130 \\
\hline 35 & 5 & 0 & 5 & 0 & 4 & 1 & 3 & 0 & 5428.7317 & -0.0045 \\
\hline 36 & 5 & 0 & 5 & 1 & 4 & 1 & 3 & 1 & 5256.6945 & 0.0230 \\
\hline 37 & 1 & 1 & 1 & 0 & 1 & 0 & 1 & 0 & 3189.0454 & 0.0019 \\
\hline 38 & 1 & 1 & 1 & 1 & 1 & 0 & 1 & 1 & 3189.6020 & 0.0130 \\
\hline 39 & 2 & 1 & 2 & 1 & 2 & 0 & 2 & 1 & 3099.9738 & -0.0095 \\
\hline 40 & 2 & 1 & 2 & 0 & 2 & 0 & 2 & 0 & 3100.7815 & -0.0079 \\
\hline
\end{tabular}


Table S4. Rotational transitions of the ${ }^{13} \mathrm{C}(1)$ isotopologue of benzyl mercaptan and differences between the observed and calculated frequencies (o-c) for the fit of Table 2 .

\begin{tabular}{ccccccccccc}
\hline & $J^{\prime}$ & $K_{-l^{\prime}}$ & $K_{+l^{\prime}}$ & $v^{\prime}$ & $J^{\prime \prime}$ & $K_{-l^{\prime \prime}}$ & $K_{+l^{\prime \prime}}$ & $v^{\prime \prime}$ & Freq. $/ \mathrm{MHz}$ & $\mathrm{o}-\mathrm{c} / \mathrm{kHz}$ \\
\hline 1 & 2 & 0 & 2 & 0 & 1 & 0 & 1 & 0 & 3779.6120 & -0.0062 \\
2 & 2 & 0 & 2 & 1 & 1 & 0 & 1 & 1 & 3762.7620 & -0.0021 \\
3 & 3 & 0 & 3 & 0 & 2 & 0 & 2 & 0 & 5662.1880 & 0.0052 \\
4 & 3 & 0 & 3 & 1 & 2 & 0 & 2 & 1 & 5632.1880 & -0.0007 \\
5 & 4 & 0 & 4 & 0 & 3 & 0 & 3 & 0 & 7536.1000 & -0.0012 \\
6 & 4 & 0 & 4 & 1 & 3 & 0 & 3 & 1 & 7485.1250 & 0.0012 \\
7 & 2 & 1 & 1 & 0 & 1 & 1 & 0 & 0 & 3886.9120 & 0.0058 \\
8 & 2 & 1 & 1 & 1 & 1 & 1 & 0 & 1 & 3885.0380 & -0.0038 \\
9 & 3 & 1 & 3 & 0 & 2 & 1 & 2 & 0 & 5533.6120 & -0.0002 \\
10 & 3 & 1 & 3 & 1 & 2 & 1 & 2 & 1 & 5507.3875 & 0.0142 \\
11 & 3 & 1 & 2 & 0 & 2 & 1 & 1 & 0 & 5824.6380 & 0.0082 \\
12 & 3 & 1 & 2 & 1 & 2 & 1 & 1 & 1 & 5827.0250 & 0.0050 \\
13 & 4 & 1 & 4 & 0 & 3 & 1 & 3 & 0 & 7383.1500 & 0.0004 \\
14 & 4 & 1 & 4 & 1 & 3 & 1 & 3 & 1 & 7353.6500 & -0.0104 \\
15 & 4 & 1 & 3 & 0 & 3 & 1 & 2 & 0 & 7749.3380 & -0.0089 \\
16 & 4 & 1 & 3 & 1 & 3 & 1 & 2 & 1 & 7767.4875 & -0.0017 \\
\hline
\end{tabular}


Table S5. Rotational transitions of the ${ }^{13} \mathrm{C}(4)$ isotopologue of benzyl mercaptan and differences between the observed and calculated frequencies (o-c) for the fit of Table 2 .

\begin{tabular}{ccccccccccc}
\hline & $J^{\prime}$ & $K_{-l^{\prime}}$ & $K_{+l^{\prime}}$ & $v^{\prime}$ & $J^{\prime \prime}$ & $K_{-l^{\prime \prime}}$ & $K_{+l^{\prime \prime}}$ & $v^{\prime \prime}$ & Freq. $/ \mathrm{MHz}$ & $\mathrm{o}-\mathrm{c} / \mathrm{kHz}$ \\
\hline 1 & 2 & 1 & 2 & 1 & 1 & 1 & 1 & 1 & 3622.7737 & -0.0087 \\
2 & 2 & 1 & 2 & 0 & 1 & 1 & 1 & 0 & 3640.1256 & -0.0099 \\
3 & 2 & 1 & 1 & 1 & 1 & 1 & 0 & 1 & 3832.4330 & -0.0001 \\
4 & 2 & 1 & 1 & 0 & 1 & 1 & 0 & 0 & 3834.2410 & 0.0042 \\
5 & 3 & 1 & 3 & 1 & 2 & 1 & 2 & 1 & 5436.3468 & 0.0147 \\
6 & 3 & 1 & 3 & 0 & 2 & 1 & 2 & 0 & 5460.9729 & -0.0022 \\
7 & 3 & 0 & 3 & 1 & 2 & 0 & 2 & 1 & 5559.4610 & -0.0162 \\
8 & 3 & 0 & 3 & 0 & 2 & 0 & 2 & 0 & 5587.6144 & 0.0039 \\
9 & 3 & 1 & 2 & 0 & 2 & 1 & 1 & 0 & 5745.9816 & 0.0066 \\
10 & 3 & 1 & 2 & 1 & 2 & 1 & 1 & 1 & 5748.1646 & 0.0039 \\
11 & 4 & 1 & 4 & 1 & 3 & 1 & 3 & 1 & 7257.9530 & -0.0074 \\
12 & 4 & 1 & 4 & 0 & 3 & 1 & 3 & 0 & 7285.5432 & 0.0063 \\
13 & 4 & 0 & 4 & 1 & 3 & 0 & 3 & 1 & 7389.9578 & 0.0085 \\
14 & 4 & 0 & 4 & 0 & 3 & 0 & 3 & 0 & 7437.3977 & -0.0009 \\
15 & 2 & 0 & 2 & 1 & 1 & 0 & 1 & 1 & 3713.7435 & 0.0085 \\
16 & 2 & 0 & 2 & 0 & 1 & 0 & 1 & 0 & 3729.6400 & -0.0017 \\
17 & 4 & 1 & 3 & 0 & 3 & 1 & 2 & 0 & 7645.7732 & -0.0077 \\
18 & 4 & 1 & 3 & 1 & 3 & 1 & 2 & 1 & 7662.4907 & -0.0030 \\
\hline
\end{tabular}


Table S6. Rotational transitions of the ${ }^{13} \mathrm{C}(\alpha)$ isotopologue of benzyl mercaptan and differences between the observed and calculated frequencies (o-c) for the fit of Table 2 .

\begin{tabular}{ccccccccccc}
\hline & $J^{\prime}$ & $K_{-l^{\prime}}$ & $K_{+1^{\prime}}$ & $v^{\prime}$ & $J^{\prime \prime}$ & $K_{-l^{\prime \prime}}$ & $K_{+l^{\prime \prime}}$ & $v^{\prime \prime}$ & Freq. $/ \mathrm{MHz}$ & $\mathrm{o-c} / \mathrm{kHz}$ \\
\hline 1 & 2 & 0 & 2 & 1 & 1 & 0 & 1 & 1 & 3747.4207 & 0.0010 \\
2 & 2 & 0 & 2 & 0 & 1 & 0 & 1 & 0 & 3762.9766 & -0.0319 \\
3 & 3 & 0 & 3 & 0 & 2 & 0 & 2 & 0 & 5637.4875 & 0.0173 \\
4 & 3 & 0 & 3 & 1 & 2 & 0 & 2 & 1 & 5609.8893 & 0.0100 \\
5 & 4 & 0 & 4 & 1 & 3 & 0 & 3 & 1 & 7456.9556 & -0.0061 \\
6 & 4 & 0 & 4 & 0 & 3 & 0 & 3 & 0 & 7503.5248 & -0.0013 \\
7 & 2 & 1 & 1 & 1 & 1 & 1 & 0 & 1 & 3866.7292 & -0.0003 \\
8 & 2 & 1 & 1 & 0 & 1 & 1 & 0 & 0 & 3868.2123 & -0.0075 \\
9 & 2 & 1 & 2 & 1 & 1 & 1 & 1 & 1 & 3655.7593 & 0.0206 \\
10 & 2 & 1 & 2 & 0 & 1 & 1 & 1 & 0 & 3672.5011 & 0.0152 \\
11 & 3 & 1 & 3 & 1 & 2 & 1 & 2 & 1 & 5486.0712 & -0.0186 \\
12 & 3 & 1 & 3 & 0 & 2 & 1 & 2 & 0 & 5509.2441 & -0.0046 \\
13 & 3 & 1 & 2 & 0 & 2 & 1 & 1 & 0 & 5796.5310 & 0.0109 \\
14 & 3 & 1 & 2 & 1 & 2 & 1 & 1 & 1 & 5799.6176 & -0.0194 \\
15 & 4 & 1 & 4 & 1 & 3 & 1 & 3 & 1 & 7325.8499 & 0.0028 \\
16 & 4 & 1 & 4 & 0 & 3 & 1 & 3 & 0 & 7349.4637 & -0.0021 \\
17 & 4 & 1 & 3 & 0 & 3 & 1 & 2 & 0 & 7711.4320 & -0.0022 \\
18 & 4 & 1 & 3 & 1 & 3 & 1 & 2 & 1 & 7731.1305 & 0.0137 \\
\hline
\end{tabular}


Table S7. Effective structure fit for gauche benzyl mercaptan and atomic coordinates.

\begin{tabular}{lcc}
\hline Fitted Parameter & $\mathrm{r}_{0}$ & Ab initio \\
\hline $\mathrm{r}(\mathrm{S}-\mathrm{C} \alpha) / \AA$ & $1.810(3)$ & 1.829 \\
$\mathrm{r}(\mathrm{C} \alpha-\mathrm{C} 1) / \AA$ & $1.490(7)$ & 1.502 \\
$\angle(\mathrm{C} 1-\mathrm{C} \alpha-\mathrm{S}) / \mathrm{deg}$ & $114.3(28)$ & 113.0 \\
$\tau(\mathrm{C} 1-\mathrm{C} \alpha-\mathrm{S}-\mathrm{H}) / \mathrm{deg}$ & $48.5(6.4)$ & 51.5 \\
$\tau(\mathrm{C} 2-\mathrm{C} 1-\mathrm{C} \alpha-\mathrm{S}) / \mathrm{deg}$ & $81.2(1.5)$ & 80.8 \\
& & \\
Derived Parameter & & \\
\hline$A / \mathrm{MHz}$ & 4167.42 & 4126.3 \\
$B / \mathrm{MHz}$ & 1001.60 & 1000.2 \\
$C / \mathrm{MHz}$ & 891.68 & 892.0 \\
$P_{b b} / \mathrm{u} \AA^{2}$ & 91.732 & 91.886 \\
\hline
\end{tabular}

\begin{tabular}{ccccccc}
\hline Atom $^{\mathrm{a}}$ & $a / \AA$ & $\sigma_{a} / \AA$ & $b / \AA$ & $\sigma_{b} / \AA$ & $c / \AA$ & $\sigma_{c} / \AA$ \\
\hline $\mathrm{S}$ & -2.61778 & 0.00075 & -0.087 & 0.010 & 0.4516 & 0.0034 \\
$\mathrm{C}$ & -1.4228 & 0.0059 & 0.187 & 0.011 & -0.8804 & 0.0025 \\
$\mathrm{C}$ & 0.00142 & 0.00053 & 0.0815 & 0.0061 & -0.45645 & 0.00027 \\
$\mathrm{C}$ & 0.7416 & 0.0076 & 1.2403 & 0.0045 & -0.178 & 0.012 \\
$\mathrm{C}$ & 2.0810 & 0.0072 & 1.15425 & 0.00061 & 0.220 & 0.012 \\
$\mathrm{C}$ & 2.68122 & 0.00033 & -0.0987 & 0.0013 & 0.3869 & 0.0012 \\
$\mathrm{C}$ & 1.9539 & 0.0074 & -1.26094 & 0.00010 & 0.098 & 0.014 \\
$\mathrm{C}$ & 0.6167 & 0.0070 & -1.1705 & 0.0040 & -0.300 & 0.013 \\
$\mathrm{H}$ & -2.024 & 0.083 & 0.742 & 0.133 & 1.315 & 0.057 \\
$\mathrm{H}$ & -1.684 & 0.080 & -0.551 & 0.099 & -1.645 & 0.062 \\
$\mathrm{H}$ & -1.569 & 0.066 & 1.183 & 0.060 & -1.305 & 0.088 \\
$\mathrm{H}$ & 0.277 & 0.013 & 2.2162 & 0.0060 & -0.308 & 0.022 \\
$\mathrm{H}$ & 2.643 & 0.013 & 2.06060 & 0.00057 & 0.431 & 0.022 \\
$\mathrm{H}$ & 3.72046 & 0.00064 & -0.1688 & 0.0043 & 0.6963 & 0.0016 \\
$\mathrm{H}$ & 2.418 & 0.013 & -2.2367 & 0.0015 & 0.214 & 0.024 \\
$\mathrm{H}$ & 0.048 & 0.013 & -2.0746 & 0.0053 & -0.506 & 0.023 \\
\hline${ }^{\circ}$ Principal inertial axes labeled as $a, b, c$, with standard deviations $\sigma_{\alpha}(\alpha=a, b, c)$.
\end{tabular}


Table S8. Predicted atomic coordinates of gauche benzyl mercaptan (MP2/6-311++G(d,p)).

\begin{tabular}{cccc}
\hline Atom & $a / \AA$ & $b / \AA$ & $c / \AA$ \\
\hline $\mathrm{S}$ & 2.6146 & 0.0935 & 0.4615 \\
$\mathrm{C}$ & -0.0045 & -0.0893 & -0.4641 \\
$\mathrm{C}$ & -0.7478 & -1.2436 & -0.1755 \\
$\mathrm{C}$ & -2.0846 & -1.1498 & 0.2297 \\
$\mathrm{C}$ & -2.6788 & 0.1065 & 0.3930 \\
$\mathrm{C}$ & -1.9484 & 1.2641 & 0.0934 \\
$\mathrm{C}$ & -0.6139 & 1.1660 & -0.3107 \\
$\mathrm{C}$ & 1.4286 & -0.2036 & -0.8989 \\
$\mathrm{H}$ & -0.2876 & -2.2220 & -0.3026 \\
$\mathrm{H}$ & -2.6487 & -2.0527 & 0.4483 \\
$\mathrm{H}$ & -3.7161 & 0.1825 & 0.7077 \\
$\mathrm{H}$ & -2.4081 & 2.2424 & 0.2068 \\
$\mathrm{H}$ & -0.0424 & 2.0667 & -0.5256 \\
$\mathrm{H}$ & 1.6639 & 0.5602 & -1.6460 \\
$\mathrm{H}$ & 1.6184 & -1.1837 & -1.3434 \\
$\mathrm{H}$ & 2.0492 & -0.7770 & 1.3035 \\
\hline
\end{tabular}

${ }^{\circ}$ Principal inertial axes labeled as $a, b, c$. 